\title{
Oral Wound Healing Effects of Acai Berry Water Extracts in Rat Oral Mucosa
}

\author{
Mi Hyun Kang and Bae-Hwan Kim \\ Major in Public Health, Faculty of Food and Health Sciences, Keimyung University, Daegu, Korea
}

\begin{abstract}
The objective of this study was to determine the oral wound healing effects of acai berry water extracts (ABWE) in rat oral mucosa. To estimate the anti-oxidative effects of $\mathrm{ABWE}$, the contents of phenolic compounds, and DPPH (1,1-diphenyl-2-picryl hydrazyl) and ABTS (2,2'-azinobis-(3-ethylbenzothiazoline-6-sulfonic acid)) abilities were evaluated. Wound healing effects of ABWE were tested following 6-day exposure after induction of wound by applying 50\% acetic acid to oral mucosa of Sprague-Dawley rats. Macroscopic and histopathological analyses were performed to determine wound healing effects of ABWE. Sodium fusidate $(20 \mathrm{mg} / \mathrm{g})$ was used as positive control. ABWE showed significantly high antioxidant effects in all assays, although its potency was weaker than the positive control. From day 3 after treatment, wound healing effects of ABWE were observed in oral mucosa. These wound healing effects were also consistent with histopathological evaluation results. Taken together, these results indicate that ABWE might have potential as an oral wound healing agent in the future.
\end{abstract}

Key words: Oral wound healing, Acai berry water extracts, Anti-oxidant, Rat oral mucosa

\section{INTRODUCTION}

Wound is an abnormal status of structure or function of the human body, and it occurs due to external damage (1). Many factors can affect the wound healing process which is very complicated phenomenon with a serial of processes (2). Because oral mucosa is always exposed to saliva and oral bacteria, delayed wound healing or scar formation with pain can occur. Many factors such as chewing and swallowing food, and pronunciation can affect wound healing of oral mucosa.

Even if size and length of the wound in the skin and mucous membrane are the same, phagocytic function and epithelial wound healing in the mucous membrane are greater than those in the skin (3). In the initial inflammation phase, blood cells such as neutrophils and monocytes

Correspondence to: Bae-Hwan Kim, Major in Public Health, Faculty of Food and Health Sciences, Keimyung University, Daegu 42601, Korea

E-mail: kim9399@kmu.ac.kr

This is an Open-Access article distributed under the terms of the Creative Commons Attribution Non-Commercial License (http:// creativecommons.org/licenses/by-nc/3.0) which permits unrestricted non-commercial use, distribution, and reproduction in any medium, provided the original work is properly cited. are gathered. They can secret growth factors (4) that play important roles in the infiltration and synthesis of collagen fibers to repair the wound (5). Fibroblasts also play an important role in wound contraction by synthesizing collagen into the connective tissue (6). Collagen is a very important component in the process of wound healing.

Various medicines such as antibiotic ointment, immunosuppressive agents, and antiseptics are usually used for the purpose of wound healing (7), but the ointment containing fusidic acid sodium or triamcinolone acetonide which are usually used for wound healing, was reported to have side effects such as dermal allergic reaction or scar formation (8), therefore natural materials without side effects are needed strongly recently.

Acai berry (Euterpe oleracea Mart.) is a tropical purple fruit with lots of anti-oxidant nutrients such as anthocyanin and polyphenols (9). It was reported that it has excellent anti-inflammation (10) and skin wound healing (11) effects. However, its wound healing effect for oral mucosal has not reported yet.

Therefore, the objective of this study was to determine the wound healing effect of acai berry water extract containing various anti-oxidant ingredients for oral mucosa using Sprague-Dawley rat model, and to estimate its potential as an oral wound healing ingredients for oral mucosal medication. 


\section{MATERIALS AND METHODS}

Reagents and preparation of acai berry water extracts (ABWE). DPPH (1,1-diphenyl-2-picryl hydrazyl), ABTS (2,2'-azinobis-(3-ethylbenzothiazoline-6-sulfonic acid)) tannic acid, azinobis acid, and Folin-Ciocalteu's phenol reagent were obtained from Sigma-Aldrich (St. Louis, MO, USA). Freeze-dried acai berry powder $(99.9 \%$ with $0.1 \%$ citric acid) was purchased from Sambazon (Paragon laboratories, MI, USA). Acai berry powder $(100 \mathrm{~g})$ was mixed with $1 \mathrm{~L}$ of water and extracted at $80^{\circ} \mathrm{C}$ for $3 \mathrm{hrs}$. Extracted substances were then filtered and freeze-dried for $72 \mathrm{hrs}$. The extraction process was repeated three times. Predominant constituents in acai berry extract such as fatty acids and amino acids (Table 1) have been reported previously (9).

\section{Antioxidant activity.}

- Total polyphenol content: Total phenol content in ABWE was measured according to Folin and Denis method (12). Briefly, distilled water $(1 \mathrm{~mL})$ was added to $5.2 \mathrm{mg}$ of $\mathrm{ABWE}$ and $0.5 \mathrm{~mL}$ of this solution was added into a test tube. Then $1.5 \mathrm{~mL}$ of phenol reagent was added, fairly mixed, and left for $5 \mathrm{~min}$. Saturated $\mathrm{Na}_{2} \mathrm{CO}_{3}(0.5 \mathrm{~mL})$ solution was then added for $1 \mathrm{hr}, \mathrm{HCl}(1 \mathrm{~N}, 0.1 \mathrm{~mL})$ was added and absorbance was measured at wavelength of 640 $\mathrm{nm}$. Total content of phenolic compounds was calculated using tannic acid as a standard.

- Electron donating ability: Electron donating ability of ABWE was measured according to the method of Blois (13). DPPH (0.2 $\mathrm{mM}$ of $1 \mathrm{~mL})$ was added into $2 \mathrm{~mL}$ of each test solution. The solution was stirred and left at room temperature for $30 \mathrm{~min}$. Absorbance of the mixture was then measured at wavelength of $517 \mathrm{~nm}$. Electron donating ability was presented by calculating the decreasing rate of absorbance for the test substance-added group and the control group.

Table 1. Nutrient analysis of freeze-dried acai berry

\begin{tabular}{lc}
\hline \hline Nutrients & Contents unit per $100 \mathrm{~g} \mathrm{DW}^{*}$ \\
\hline Calories & 533.9 \\
Calories from fat & 292.6 \\
Total fat & $32.5 \mathrm{~g}$ \\
Saturated fat & $8.1 \mathrm{~g}$ \\
Cholesterol & $13.5 \mathrm{mg}$ \\
Sodium & $30.4 \mathrm{mg}$ \\
Total carbohydrate & $52.2 \mathrm{~g}$ \\
Dietary fibre & $44.2 \mathrm{~g}$ \\
Sugars & $1.3 \mathrm{~g}$ \\
Protein & $8.1 \mathrm{~g}$ \\
Vitamin A & $1002 \mathrm{IU}$ \\
Vitamin C & $<0.1 \mathrm{mg}$ \\
Calcium & $260.0 \mathrm{mg}$ \\
Iron & $4.4 \mathrm{mg}$ \\
\hline
\end{tabular}

"DW: Dry weight.
Donating ability $(\%)=\left[1-\left(\mathrm{A}_{\text {sample }} / \mathrm{A}_{\text {control }}\right)\right] \times 100$

where $\mathrm{A}_{\text {control }}$ is the absorbance of the control (i.e., reagents without test substance).

- ABTS radical scavenging ability: Free radical scavenging ability was investigated using ABTS cation decolorization assay (14). $\mathrm{ABTS}^{+}$was formed by mixing $7 \mathrm{mM}$ 2,2'-azinobis-(3-ethylbenzothiazoline-6-sulfonic acid) and $2.4 \mathrm{mM}$ potassium persulfate (final concentration). It was left at room temperature in a dark place for $12 \mathrm{hrs}$. The mixture was then diluted with ethanol to an absorbance of $0.7 \pm 0.02$ at $734 \mathrm{~nm}$. Sample $(100 \mathrm{~mL})$ diluted at each concentration was then mixed with $100 \mathrm{~mL}$ of ABTS solution which was left at room temperature in a darkroom for $7 \mathrm{~min}$. The absorbance was then measured at wavelength of $734 \mathrm{~nm}$. Radical scavenging ability of each extract was calculated using the following formula (in comparison with control group without addition of extract):

$$
\text { Scavenging ability }(\%)=\left[1-\left(\mathrm{A}_{\text {sample }} / \mathrm{A}_{\text {control }}\right)\right] \times 100
$$

- Superoxide dismutase (SOD)-like activity: SODlike activity ability was measured using the method of Marklund and Marklund (15). Briefly, the sample (0.2 $\mathrm{mL}$ ) was mixed with $2.6 \mathrm{~mL}$ of Tris-HCl buffer mixture $(50 \mathrm{mM}$ tris and $10 \mathrm{mM}$ EDTA, $\mathrm{pH} 8.5)$ and incubated at $25^{\circ} \mathrm{C}$ for $10 \mathrm{~min}$. The reaction was then stopped by adding $0.1 \mathrm{~mL}$ of $1 \mathrm{~N} \mathrm{HCl}$. Absorbance was measured at wavelength of $420 \mathrm{~nm}$. SOD-like activity was calculated using the following formula (in comparison with control group without addition of extract):

$$
\text { SOD-like activity }(\%)=\left[1-\left(\mathrm{A}_{\text {sample }} / \mathrm{A}_{\text {control }}\right)\right] \times 100
$$

\section{In vivo wound healing experiment.}

- Animals: Male Sprague Dawley (SD) rats (6-weekold, 190 210 g) were purchased from Daehan biolink (Eumsung, Chungbuk) and acclimatized for one week, and then assigned to each group. The animal room was maintained at temperature of $22 \pm 3^{\circ} \mathrm{C}$ with relative humidity of $50 \pm 10 \%$ with $12 \mathrm{hr} / 12 \mathrm{hr}$ of light/dark cycle per day. They were provided free access to feed (Purina, Korea) and water ad libitum during the experiment period. The experimental protocol was approved by the Institutional Animal Care and Use Committee (IACUC) of Keimyung University.

- Design of oral mucosal wound model. The method of Fujisawa et al. (16) was adopted for generating oral mucosa wound model. A cotton ball of $6 \mathrm{~mm}$ in diameter was soaked with $50 \%$ acetic acid and a wound was induced by contacting it to the lower anterior part of gingiva for $2 \mathrm{~min}$. Test material (ABWE, $50 \mu \mathrm{L}$ ) was applied to the wounded area once daily for 6 days until complete epithelialization. 
Table 2. Experiment groups

\begin{tabular}{llc}
\hline \hline Groups & Test compound & $\begin{array}{c}\text { No. } \\
\text { of rats }\end{array}$ \\
\hline Vehicle Control (VC) & $\begin{array}{c}\text { 2\% Carboxymethyl cellulose (CMC) } \\
\text { in distilled water }\end{array}$ & 5 \\
Positive Control (PC) & 2\% Sodium fusidate & 5 \\
Experimental 1 (E1) & 1\% ABWE in 2\% CMC & 5 \\
Experimental 2 (E2) & 3\% ABWE in 2\% CMC & 5 \\
Experimental 3 (E3) & $5 \%$ ABWE in 2\% CMC & 5 \\
\hline
\end{tabular}

ABWE: Acai berry water extract.

- Constitution of the experimental group: Five animals were assigned to each group as follows by randomized block design after induction of mucosal wound. Vehicle control group (VC) that was treated with $2 \%$ of carboxymethyl cellulose (CMC); Positive control group (PC) that was treated with $150 \mathrm{mg}$ of ointment purchased from the market including $20 \mathrm{mg} / \mathrm{g}$ of sodium fusidate; The group applied by $1 \%$ ABWE (E1), the group applied by $3 \%$ ABWE (E2) and the group applied by 5\% ABWE (E3) (Table 2).

- Macroscopic observation: Water intake, feed intake, body weight and feed efficiency ratio were measured daily (10 o'clock in the morning) throughout the study period.

To observe the wound with naked eyes, photos of dermal wounds were taken using a digital camera at 0,3 , and 6 days before applying test materials for each group. Wound areas were graded subjectively as no lesion (-), mild $(+)$, moderate $(++)$ and severe $(+++)$ for epidermal or dermal restoration.

- Histopathological observation: After the experiment was finished, mucosal tissue of the wounded area was fixed in neutral formalin solution $(10 \%)$ for $24 \mathrm{hrs}$ and embedded in paraffin with routine process. The embedded tissue was cut into pieces ( $4 \mu \mathrm{m}$ in thickness) and stained with hematoxylin and eosin (H\&E) to observe changes in dermal tissue such as thickness of epidermis and permeation of inflammatory cells. They were also stained with toluidine blue to observe the distribution of mast cells. Sections were also stained with Masson's trichrome to observe changes in dermal collagen using an optical microscope.

- Statistical analysis: Data are presented as mean \pm SD. Statistically significant differences between groups were determined by one-way analysis of variance (ANOVA) followed by Duncan's multiple-range test at $p<0.05$ using SPSS 21.0 for windows (IBM, Armonk, NY, USA).

\section{RESULTS}

Antioxidant activity of ABWE. The extraction yield of ABWE was $17.31 \pm 1.06 \%$. Total polyphenol content in ABWE was $43.0 \pm 1.82 \mathrm{mg} / \mathrm{g}$ using tannic acid as standard. Electron donating ability of ABWE at 1,000 ppm
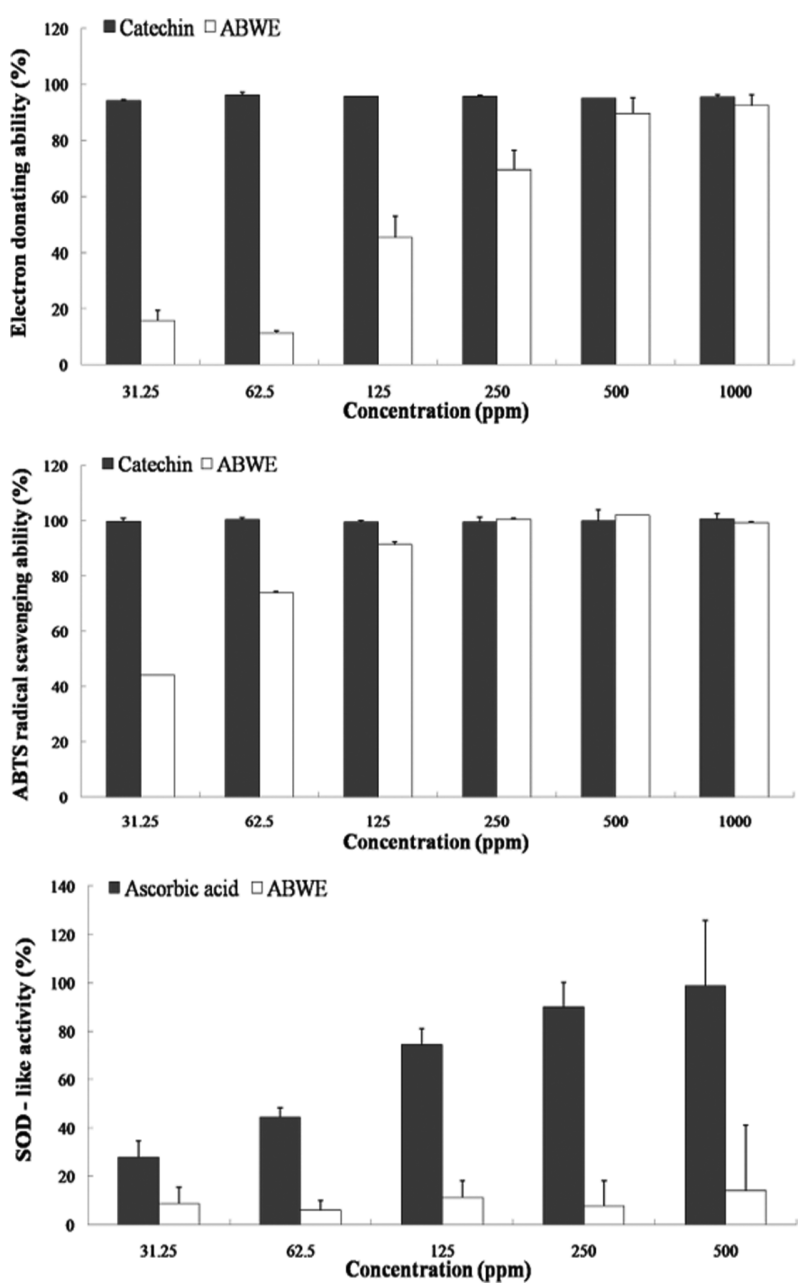

Fig. 1. Electron donating ability, ABTS radical scavenging ability and SOD-like activity of ABWE. Values represent the mean \pm SD of 3 independent measurements. ABWE: Acai berry water extract.

was $92.5 \pm 3.11 \%$. This value was slightly lower than that $(95.5 \%)$ of positive control catechin at the same concentration. ABTS radical scavenging ability of ABWE at $1,000 \mathrm{ppm}$ was $99.4 \%$. This value was also slightly lower than that $(100.6 \%)$ of the positive control catechin at the same concentration. SOD-like activity of ABWE at 500 ppm was $14 \%$. This value was lower than vitamin $\mathrm{C}$ (ascorbic acid) as the positive control (Fig. 1).

Macroscopic observation of rat oral wounds. Water intake, feed intake, body weight, or feed efficiency ratio was not significantly different between groups (data not shown). Each group displayed ulcer and acute inflammatory changes, excreting exudation around the mucosal wound. Wound size was observed to be restored from day 3 after wound was made. Wound area become smaller and completed epithelization with rare excretion in ABWE treated group at day 6 after the wound was made compared 


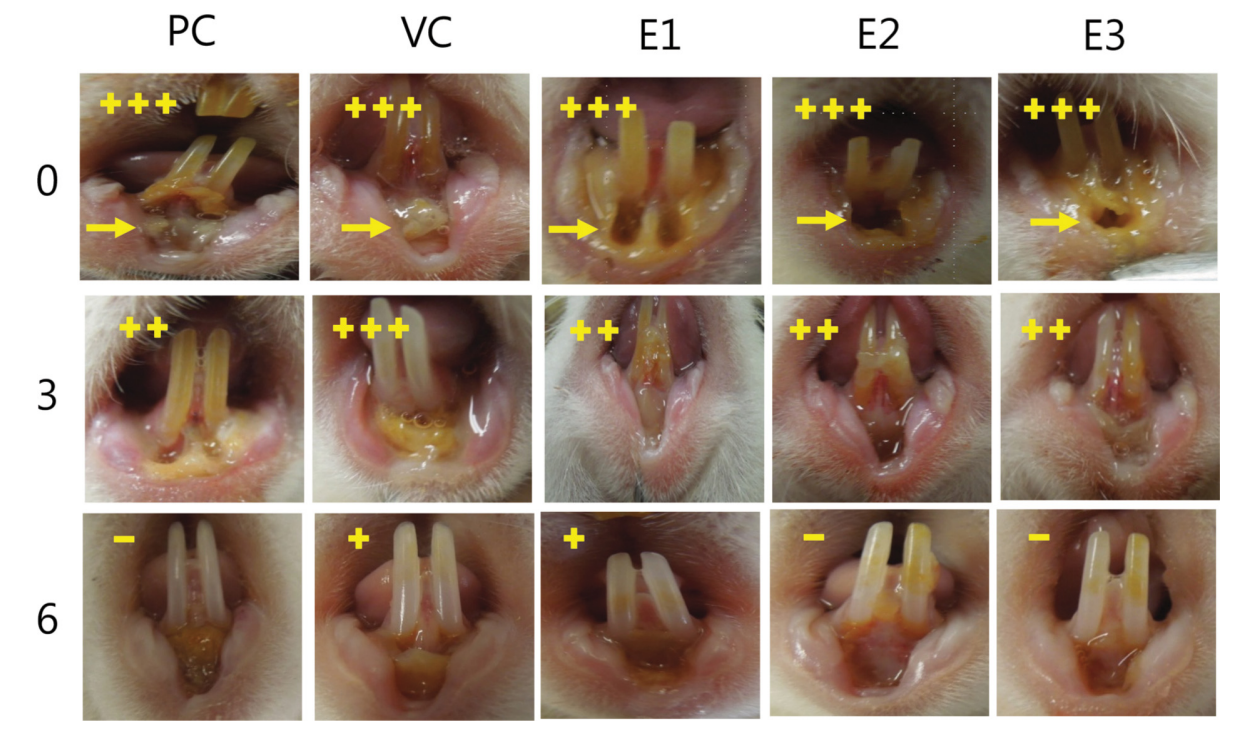

Fig. 2. Macroscopic observations of wounds at the oral mucosa. Differences of the healing areas in PC, VC, E1, E2, and E3 groups ( 5 animals per group) at the oral wounds. Severe wound tissues (+++, arrow) were restored by applying of ABWE at day 3 and day 6. PC: $2 \%$ sodium fusidate, VC: $2 \%$ carboxymethyl cellulose (CMC), E1: 1\% ABWE, E2: $3 \%$ ABWE, E3: 5\% ABWE. ABWE: Acai berry water extract.

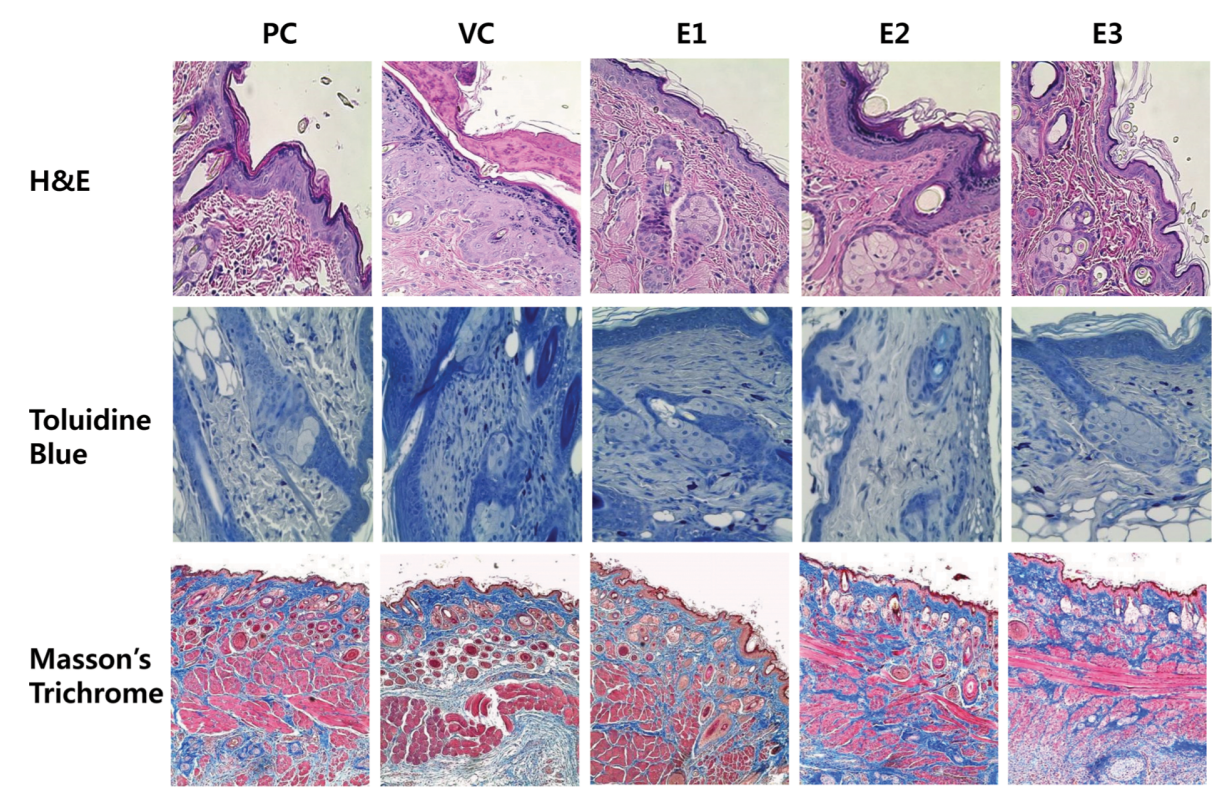

Fig. 3. Microscopical examination of oral mucosa $(\times 100)$. Histological appearance of wounds from experimental groups $(5$ animals per group) by $\mathrm{H} \& \mathrm{E}$, toluidine blue and masson's trichrome staining on day 6 on oral mucosa after wounding. PC: $2 \%$ sodium fusidate, VC: 2\% carboxymethyl cellulose (CMC), E1: 1\% ABWE, E2: 3\% ABWE, E3: 5\% ABWE. ABWE: Acai berry water extract.

to that of the vehicle control group. There was much less scar in E2 or E3 group compared to that in the VC (Fig. 2).

Histopathological observation of oral mucosa. Inflammation was recovered in oral mucosa of ABWE applied group compared to that in the vehicle control group based on H\&E staining. The number of mast cells was diminished in the group applied with ABWE compared to that in the vehicle control group based on toluidine blue staining. Higher concentration of collagen was shown in ABWE applied group compared to that in the vehicle control group based on Masson's trichrome staining (Fig. 3).

\section{DISCUSSION}

There have been many studies on the development of 
natural medicine without side effect, such as aucuba (17), Noni (18), chamomile (19) and Lespedeza cuneata (20). Especially, antioxidants have been reported to be effective in wound healing because they can induce skin regeneration by protecting cells or organisms from damage (21). Anthocyanin, proanthocyanidin, flavonoids, and lignan are known to be main polyphenols in acai berry (22). This study showed that water extract of acai berry also had oral wound healing effect. This might be associated with its high antioxidant activity. Total polyphenol content of acai berry has been reported to be higher in water extract than that in ethanol extract (23).

The oral wound healing effect of ABWE was assessed by measuring the wound area. Histopathological changes were also observed in rat oral mucosa to confirm the wound healing process. The group treated with ABWE had significantly reduced wound area compared to the control group since day 3 after creating the wound. On day 6 after creating the wound in oral mucosa, the group treated with ABWE also showed re-epithelization. The ABWE treated group showed less inflammatory lesion in H\&E staining, increased collagen contents in Masson's trichrome staining, and decreased mast cells in Toluidine blue staining compared to the control group. Mast cells play an important role in increasing inflammation by secreting histamine. Thus, decrease of mast cells can help improve wound healing by decreasing inflammation (24). In the present study, the group treated with ABWE displayed fastest regeneration of tissue. Permeation of production of collagen might have enhanced the speed of wound healing in oral mucosa as in the skin reported previously (11). Diminished immune cells might have also reduced the inflammation of oral mucosa. Wound healing begins from inflammation right after a wound is inflicted. Suppressed inflammation and faster reepithelization will lead to quicker wound contraction (25). Results of this study showed that ABWE could prevent inflammation of the wound in oral mucosa and promote re-epithelization. In addition, ABWE could help mucosal regeneration and collagen synthesis at time of remodeling. This finding seems to be similar to study results of natural materials that promote wound healing (26).

In summary, this study confirms that ABWE has potential to be developed as oral wound healing material. However, additional studies are needed to determine the main action mechanism involved in its effect. Clinical trials are also needed in the future before ABWE can be used as medication for oral wound healing.

Received December 13, 2017; Revised February 2, 2018; Accepted February 26, 2018

\section{REFERENCES}

1. Muralidhar, A., Babu, K.S., Sankar, T.R., Reddanna, P. and
Latha, J. (2011) Evaluation of wound healing properties of bioactive fractions from the extract of Butea monosperma (lam) stem bark. Int. J. Phytomed., 3, 41-49.

2. Velnar, T., Bailey, T. and Smrkolj, V. (2009) The wound healing process: an overview of the cellular and molecular mechanisms. J. Int. Med. Res., 37, 1528-1542.

3. Sciubba, J.J., Waterhouse, J.P. and Meyer, J. (1978) A fine structural comparison of the healing of incisional wounds of mucosa and skin. J. Oral Pathol., 7, 214-227.

4. Kirsner, R.S. and Eaglstein, W.H. (1993) The wound healing process. Dermatol. Clin., 11, 629-640.

5. Park, J.E. and Barbul, A. (2004) Understanding the role of immune regulation in wound healing. Am. J. Surg., 187, 11S-16S.

6. Diegelmann, R.F. and Evans, M.C. (2004) Wound healing: an overview of acute, fibrotic and delayed healing. Front. Biosci., 9, 283-289.

7. Cleland, W.P., Jr. (2001) Opportunities and obstacles in veterinary dental drug delivery. Adv. Drug Deliv. Rev., 50, 261275.

8. Han, D.O., Lee, H.J. and Hahm, D.H. (2009) Wound-healing activity of Astragali radix in rats. Methods Find. Exp. Clin. Pharmacol., 31, 95-100.

9. Schauss, A.G., Wu, X., Prior, R.L., Ou, B., Patel, D., Huang, D. and Kababick, J.P. (2006) Phytochemical and nutrient composition of the freeze-dried amazonian palm berry, Euterpe oleraceae mart. (acai). J. Agric. Food Chem., 54, 8598-8603.

10. Kim, S.Y., Park, K.H., Moon, H.D., Jung, S.H., Lee, S.E. and Kim, B.H. (2017) Anti-inflammatory effects of acai berry ethanol extracts. J. Kor. Soc. Cosm., 23, 669-676.

11. Kang, M.H., Choi, S.H. and Kim, B.H. (2017) Skin wound healing effects and action mechanism of acai berry water extracts. Toxicol. Res., 33, 149-156.

12. Folin, O. and Denis, W. (1912) On phosphotungstic-phosphomolybdic compounds as color regents. J. Biol. Chem., 12, 239-243.

13. Blois, M.S. (1958) Antioxidant determinations by the use of a stable free radical. Nature, 181, 1199-1200.

14. Re, R., Pellegrini, N., Proteggente, A., Pannala, A., Yang, M. and Rice-Evans, C. (1999) Antioxidant activity applying an improved ABTS radical cation decolorization assay. Free Radic. Biol. Med., 26, 1231-1237.

15. Marklund, S. and Marklund, G. (1974) Involvement of the superoxide anion radical in the autoxidation of pyrogallol and a convenient assay for superoxide dismutase. Eur. $J$. Biochem., 47, 469-474.

16. Fujisawa, K., Miyamoto, Y. and Nagayama, M. (2003) Basic fibroblast growth factor and epidermal growth factor reverse impaired ulcer healing of the rabbit oral mucosa. J. Oral Pathol. Med., 32, 358-366.

17. Shim, K.M., Kim, S.E., Choi, J.Y., Park, J.C., Jeong, S.J., Lee, J.Y., Bae, C.S., Park, D.H., Kim, D.M. and Jeong, M.J. (2006) Effects of Aucuba japonica extract on oral wound healing. J. Vet. Clin., 23, 55-60.

18. Nayak, B.S., Sandiford, S. and Maxwell, A. (2009) Evaluation of the wound healing activity of ethanolic extract of Morinda citrifolia L. leaf. Evid. Based Complement. Alternat. Med., 6, 351-356.

19. Duarte, C.M., Quirino, M.R., Patrocínio, M.C. and Anbinder, 
A.L. (2011) Effects of Chamomilla recutita (L.) on oral wound healing in rats. Med. Oral Patol. Oral Cir. Bucal, 16, 716-721.

20. Jung, H.K., Kim, K.S. and Jeong, Y.S. (2014) Wound healing effects of Lespedeza cuneate extract. J. Korea Soc. Food Sci. Nutr., 43, 374-380.

21. Sen, C.K., Khanna, S., Gordillo, G., Bagchi, D., Bagchi, M. and Roy, S. (2002) Oxygen, oxidants, and antioxidants in wound healing: an emerging paradigm. Ann. N. Y. Acad. Sci., 957, 239-249.

22. Kang, J., Li, Z., Wu, T., Jensen, G.S., Schauss, A.G. and Wu, X. (2010) Anti-oxidant capacities of flavonoid compounds isolated from acai pulp (Euterpe oleracea Mart.). Food Chem., 122, 610-617.

23. Kim, J.Y., Hong, J.E., Jung, H.K., Jeong, Y.S. and Cho, K.H. (2012) Grape skin and loquat leaf extracts and acai puree have potent anti-atherosclerotic and anti-diabetic activity in vitro and in vivo in hypercholesterolemic zebrafish. Int. J. Mol. Med., 30, 606-614.

24. Lee, N.Y., Chung, K.S., Jin, J.S., Lee, Y.C. and An, H.J. (2017) The inhibitory effect of nodakenin on mast-cellmediated allergic inflammation via downregulation of NF$\kappa \mathrm{B}$ and caspase-1 activation. J. Cell Biochem., 118, 39934001.

25. Clark, R.A. (1993) Regulation of fibroplasia in cutaneous wound repair. Am. J. Med. Sci., 306, 42-48.

26. Buranasukhon, W., Athikomkulchai, S., Tadtong, S. and Chittasupho, C. (2017) Wound healing activity of Pluchea indica leaf extract in oral mucosal cell line and oral spray formulation containing nanoparticles of the extract. Pharm Biol., 55, 1767-1774. 